Electronic Physician (ISSN: 2008-5842)

http://www.ephysician.ir

December 2017, Volume: 9, Issue: 12, Pages: 5974-5984, DOI: http://dx.doi.org/10.19082/5974

\title{
A fuzzy rule-based expert system for diagnosing cystic fibrosis
}

Maryam Hassanzad $^{1}$, Azam Orooji ${ }^{2}$, Ali Valinejadi ${ }^{3}$, Aliakbar Velayati ${ }^{4}$

${ }^{1}$ M.D., Associate Professor, Pediatric Respiratory Disease Research Center, National Research Institute of Tuberculosis and Lung Diseases (NRITLD), Masih Daneshvari Hospital, Shahid Beheshti University of Medical Sciences, Tehran, Iran

${ }^{2}$ Ph.D. Candidate of Medical Informatics, Department of Health Information Management and Technology, School of Health Management and Information Sciences, Iran University of Medical Sciences, Tehran, Iran

${ }^{3}$ Ph.D. of Health Information Management, Assistant Professor, Social Determinants of Health Research Center, Department of Health Information Technology, Semnan University of Medical Sciences, Semnan, Iran

${ }^{4}$ M.D., Distinguished Professor, Mycobacteriology Research Center, National Research Institute of Tuberculosis and Lung Diseases (NRITLD), Masih Daneshvari Hospital, Shahid Beheshti University of Medical Sciences, Tehran, Iran

\section{Type of article: Original}

\begin{abstract}
Background: Finding a valid diagnosis is mostly a prolonged process. Current advances in the sector of artificial intelligence have led to the appearance of expert systems that enrich the experiences and capabilities of doctors for making decisions for their patients.

Objective: The objective of this research was developing a fuzzy expert system for diagnosing Cystic Fibrosis (CF).

Methods: Defining the risk factors and then, designing the fuzzy expert system for diagnosis of CF were carried out in this cross-sectional study. To evaluate the performance of the proposed system, a dataset that corresponded to 70 patients with respiratory disease who were serially admitted to the CF Clinic in the Pediatric Respiratory Diseases Center, Masih Daneshvari Hospital in Tehran, Iran during August 2016 to January 2017 was considered. Whole procedures of system construction were implemented in a MATLAB environment.

Results: Results showed that the suggested system can be used as a strong diagnostic tool with $93.02 \%$ precision, $89.29 \%$ specificity, $95.24 \%$ sensitivity and $92.86 \%$ accuracy for diagnosing CF. There was also a good relationship between the user and the system through the appealing user interface.

Conclusion: The system is equipped with information, knowledge, and expertise from certified specialists; hence, as a training tool it can be useful for new physicians. It is worth mentioning that the accomplishment of this project depends on advocacy of decision making in CF diagnosis. Nevertheless, it is expected that the system will reduce the number of false positives and false negatives in unusual cases.

Keywords: Expert systems, Fuzzy logic, Cystic fibrosis
\end{abstract}

\section{Introduction}

Providing accurate diagnosis and related treatment is arduous especially in cases of multiple differential diagnoses. Accordingly, finding the valid diagnosis is a lengthy process (1). Diagnosis and management of diseases are indeed a crucial task that cannot be acquired just from textbooks or classroom knowledge. It has to be acquired gradually through years of observation and experience. Moreover, most clinical cases are full of ambiguities altering in degree. During assessment, the patients portray their symptoms using adverbs such as "never, rarely, sometimes, often, mostly, and always" and each specific symptom may appear as "mild, moderate, or severe". This reflects the fact that almost

\section{Corresponding author:}

Dr. Ali Valinejadi, Social Determinants of Health Research Center, Department of Health Information Technology, Semnan University of Medical Sciences, Semnan, Iran.

Tel: +98.2333614664, Fax: +98.2333614664, Email: valinejadi.a@semums.ac.ir

Received: August 09, 2017, Accepted: November 27, 2017, Published: December 2017

iThenticate screening: November 28, 2017, English editing: December 09, 2017, Quality control: December 15, 2017 This article has been reviewed / commented by four experts

(C) 2017 The Authors. This is an open access article under the terms of the Creative Commons Attribution-NonCommercialNoDerivs License, which permits use and distribution in any medium, provided the original work is properly cited, the use is noncommercial and no modifications or adaptations are made. 
all the symptoms are experienced and described differently by each patient. Therefore, medical problems cannot be generalized or analyzed by using binary logic including "yes" or "no" options. So, an analytical program is needed. The best answer is utilizing fuzzy logic that has the capability of integrating human heuristics into computer-assisted decision making (2). Cystic fibrosis (CF) is one of the diseases that needs to consider multiple differential diagnoses; and finding accurate diagnosis is frequently an extended process. Based on the history of patients, the head (A.K.) of the CF referral center in Iran, declared that under-diagnosis of CF in Iran is significant. The carrier frequency of $2.5 \%$ (1 in 40) was defined from the frequency of heterozygous patients. It affirms that contrary to popular belief, CF might be a prevalent underdiagnosed disease in Iran. The median survival age in economically developed countries is about 40 years but it is significantly less in developing countries, such as Iran, where the incidence of CF has not been critically assessed (3). The elements that might be have participated to the broad domain of CF incidence published from Asian countries include (but are not limited to) under-diagnosis, under-reporting, deficiency of national registries, and mutability in the frequency of CF mutation carriers (4). Although there are no precise data about the prevalence of CF in different parts of Iran, the disease appears not to be rare in this country (5-7). Different Iranian populations originating from various geographical and ethnic groups, all with polymorphism of the CFTR gene, give rise to diversity of CFTR mutations in frequency and distribution (8-11). New attainments in the field of artificial intelligence have provided expert systems for medical applications. Moreover, in the last few decades computational tools have been designed to improve the experiences and abilities of physicians to find the optimal diagnosis. Expert system (ES) is an intelligent computer system including an orderly knowledge structure that emulates the experts' problem-solving skills to make complicated decisions in a specific field. For example, expert systems in medical applications are being used for diagnosis. All the patient's data and symptoms are fed into the system, which then processes the information into its possible diagnosis along with the suggested treatment and drugs prescription (12, 13). Rule-based expert systems are the most recognized of all knowledge-based systems. Knowledge is displayed in a structured arrangement of IF-THEN guidelines. Decision making in medical diagnosis is contingent on the experience, expertise and perception of the physician. Existence of more complications in the system will lead to more vagueness and doubt. Fuzzy logic has brought considerable change in eliminating ambiguity and uncertainty. Interpretation of information is based on the compositional rule of fuzzy inference. Besides, expert human knowledge is essential to specify the parameters. In such systems, the accumulated data from the domain experts should be transferred to knowledge and it should be used at the true time (12). Previous studies suggest that fuzzy relations should be considered as a good method to provide valid diagnoses and decision-making procedures in pediatrics. Therefore, expert knowledge can be utilized in the form of a fuzzy expert system in tracing and diagnosis of CF. It is suggested that utilizing this method, combined with the clinical tools for diagnosis of various diseases and conditions, may greatly decrease incorrect diagnoses. The fuzzy expert approach is more effective than machine-learning practices (14). Tables 1,2 depict the main expert systems that were developed for diagnosis purposes in various diseases and respiratory system diseases respectively. The objective of present study was developing an automated fuzzy expert system for diagnosing Cystic Fibrosis (CF) using the information related to risk factors and symptoms of this disease.

Table 1. The main expert systems that were developed for diagnosis purposes in different diseases

\begin{tabular}{|l|l|l|}
\hline $\begin{array}{l}\text { Ref. } \\
\text { no }\end{array}$ & Year & Developed/ Proposed/ Designed/ Applied/ Evaluated/ System \\
\hline 15 & 2004 & a fuzzy expert system in the prediction of neonatal resuscitation \\
\hline 16 & 2008 & a fuzzy system for diagnosis of liver disorders \\
\hline 17 & 2009 & a systematic Type-II fuzzy expert system for diagnosing human brain tumors \\
\hline 18 & 2011 & a fuzzy expert system for the control of glycemia in type 1 diabetic patients \\
\hline 19 & 2011 & an expert system to assist dentists in treating mobile teeth \\
\hline 20 & 2011 & $\begin{array}{l}\text { an expert system for diagnosis of breast cancer in order to differentiate between benign and } \\
\text { malignant breast cancer based on neuro-fuzzy rules }\end{array}$ \\
\hline 21 & 2012 & a fuzzy system to learn, analyze and diagnose heart disease \\
\hline 2 & 2013 & a fuzzy expert system designed using MATLAB is proposed for identification of severity of CTS \\
\hline 22 & 2013 & an expert system for diagnosis of cervical neoplasia (CN) precursor injuries \\
\hline 23 & 2014 & a fuzzy expert system for spinal cord disorders \\
\hline 24 & 2014 & a fuzzy rule-based expert system for Multiple Sclerosis (MS) diagnosis \\
\hline 25 & 2015 & a type-2 fuzzy system that is diagnosing and differentiating Astrocytomas in MRI scans \\
\hline
\end{tabular}


http://www.ephysician.ir

Table 2. The main expert systems that were developed for diagnosis purposes in respiratory system diseases

\begin{tabular}{|l|l|l|}
\hline $\begin{array}{l}\text { Ref. } \\
\text { no }\end{array}$ & Year & Developed/ Proposed/ Designed/ Applied/ Evaluated/ System \\
\hline 26 & 2009 & a computer-aided intelligent diagnostic system for bronchial asthma \\
\hline 27 & 2010 & a fuzzy rule-based expert system for diagnosing asthma at initial stages \\
\hline 28 & 2010 & improvised (27) for evaluating the possibility of fatal asthma \\
\hline 29 & 2011 & $\begin{array}{l}\text { a computerized clinical decision support system, designed by pediatric pulmonologists for } \\
\text { asthma }\end{array}$ \\
\hline 30 & 2012 & an expert diagnostic system for diagnosis of lung cancer \\
\hline 31 & 2012 & a fuzzy rule-based system for evaluating level of asthma exacerbation \\
\hline 32 & 2012 & an asthma management system for a pediatric emergency department \\
\hline 33 & 2012 & a fuzzy rule-based expert system for assessment severity of asthma \\
\hline 34 & 2013 & a system patient-centered computer application system for diagnosing pediatric asthma \\
\hline 35 & 2013 & $\begin{array}{l}\text { a fuzzy expert system that takes into account details of various patients, primarily with asthma } \\
\text { and Chronic Obstructive Pulmonary Disease (COPD) }\end{array}$ \\
\hline 12 & 2015 & a fuzzy rule-based medical expert system for diagnosis of lung cancer \\
\hline 36 & 2015 & $\begin{array}{l}\text { a computer-aided classification method using computed tomography (CT) images of the lung- } \\
\text { based ensemble of three classifiers including MLP, KNN and SVM }\end{array}$ \\
\hline
\end{tabular}

\section{Material and Methods}

This research is a cross-sectional study conducted in three steps:

\subsection{Definition of risk factors}

A list of 50 substantial CF risk factors was found in medical literature and they were organized in a questionnaire. Then, three domain experts were asked to assign suitable weight between 1 (non-significant) to 5 (very significant) to the factors based on their significance in $\mathrm{CF}$ diagnosis. According to the completed questionnaires, 10 parameters with the highest weight were selected.

\subsection{Designing Fuzzy Expert System}

In order to diagnose Cystic Fibrosis (CF), fuzzy set theory was applied in this survey. A rule-based fuzzy expert system (FES) has been developed. It utilized the selected factors in the first step and simulated a specialist behavior to diagnose CF. Fuzzy expert systems are basically knowledge-based systems using Fuzzy logic, Fuzzy IF-THEN rules and Membership Functions (MF) (37). Figure 1 illustrates the common architecture of a fuzzy expert system displaying the flow of data throughout the system (38). Its basic structure entails four main components:

- A fuzzifier that interprets crisp input (classical numbers) into fuzzy values;

- An inference engine that uses a fuzzy reasoning function to take a fuzzy output (in the case of Mamdani inference);

- A knowledge base that includes a set of fuzzy rules and a set of membership functions displaying the fuzzy sets of linguistic variables;

- A defuzzifier that interprets fuzzy output into crisp values (39).

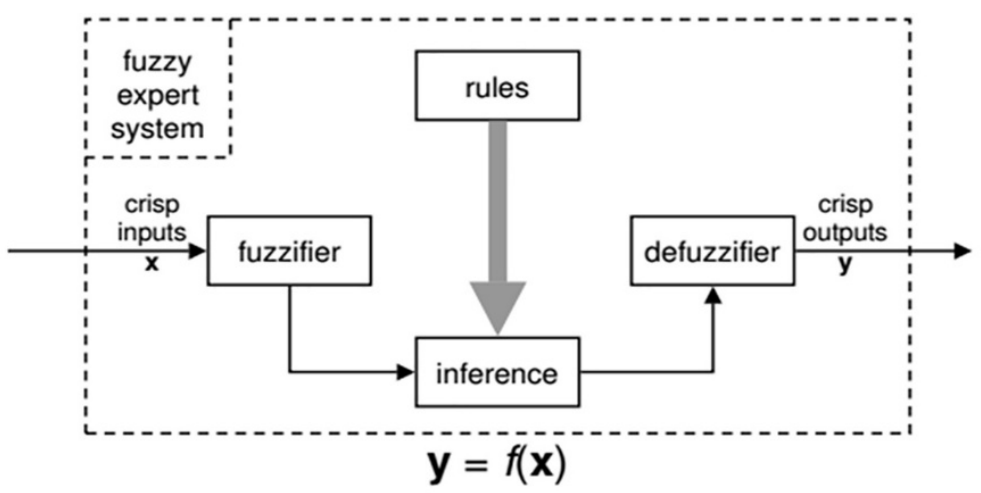

Figure 1. Common architecture of a fuzzy expert system (38) 
The first step of designing a fuzzy expert system is to determine input and output variables (40). There are 10 input variables (including Sweat test, PH, BMI, Sputum, Dyspnea, Cough, Pseudomonas, Staphylococcus, Family history, and Abnormal stool) and 1 output variable (CF diagnosis). Secondly, the membership functions of all variables have to be designed. These membership functions specify the membership of objects to fuzzy sets. Membership functions were used for input variables according to both the literature review and domain expert opinions. The detailed description of input variables, fuzzy linguistic sets, and membership functions are displayed in Table 3 . The decision process is done by the inference engine while using rules contained in the knowledge base. Knowledge base is the principal section in the fuzzy inference system that the performance of fuzzy system is dependent on its rules (40). These fuzzy rules determine the relation between fuzzy input and output. The formula to a fuzzy rule is; if antecedent, then consequent. Fuzzy operators express the antecedent, and the outcome is an expression that administers fuzzy values to the output variables. Since there were lots of input variables in this study, combining all possible inputs led to the construction of so many rules (for this paper about $2^{\wedge} 10$ ). However, in order to increase efficiency and reduce complications, only relevant rules were considered based on expert opinion. The knowledge used in the proposed system was collected based on the experts' interviews and other scientific references such as books and websites. Moreover, the Mamdani min-max approach was adopted for the inference mechanism. First, input variables were compared with the membership functions of the antecedent part in obtaining the membership values of each linguistic term. Secondly, the membership values of the antecedent part were combined to deduce firing strength of each rule, and then the consequence or results of each rule were generated. Finally, the results or consequences were aggregated to produce a crisp value by a process called defuzzification. The crisp output was calculated by the center of gravity (COG) defuzzifier (41) by the following formula:

$$
y^{*}=\frac{y \mu_{B^{\prime}} y d y}{\mu_{B^{\prime}} y d y}
$$

Where the $\mathrm{y}^{*}$ is the center of the area covered by the membership function of $B^{\prime}$. Figure 2 illustrates the fuzzy inference system block diagram created for CF diagnosis with ten inputs and one output. Additionally, Figure 3 represents the constructed FIS system characteristics that are adjusted by user. MATLAB R2014a software was used to build the fuzzy model and to increase the efficiency and user-friendliness of the system; user interface was designed by Visual Studio and C\# programming language. Figure 4 displays the graphical user interface (GUI) of the suggested system.

Table 3. Detailed description of input variables, fuzzy linguistic sets, and membership functions

\begin{tabular}{|c|c|c|c|c|c|}
\hline Type & No. & Variable name & Linguistic set & Actual Range of Variable & Type of Membership Function \\
\hline \multirow[t]{22}{*}{ Input } & \multirow[t]{2}{*}{1} & \multirow[t]{2}{*}{ Sweat test } & Low & $<60$ & Sigmoidal \\
\hline & & & High & $>60$ & Sigmoidal \\
\hline & \multirow[t]{3}{*}{2} & \multirow[t]{3}{*}{$\mathrm{PH}$} & Low & $<7.35$ & Gaussian \\
\hline & & & Normal & $>7.35$ and $<7.45$ & Gaussian \\
\hline & & & High & $>7.45$ & Gaussian \\
\hline & \multirow[t]{3}{*}{3} & \multirow[t]{3}{*}{ BMI } & Underweight & $<18$ & Gaussian \\
\hline & & & Healthy & $>18$ and $<25$ & Gaussian \\
\hline & & & Overweight & $>25$ & Gaussian \\
\hline & \multirow[t]{2}{*}{4} & \multirow[t]{2}{*}{ Sputum } & Negative & 1 & Singleton \\
\hline & & & Positive & 2 & Singleton \\
\hline & \multirow[t]{2}{*}{5} & \multirow[t]{2}{*}{ Dyspnea } & Negative & 1 & Singleton \\
\hline & & & Positive & 2 & Singleton \\
\hline & \multirow[t]{2}{*}{6} & \multirow[t]{2}{*}{ Cough } & Negative & 1 & Singleton \\
\hline & & & Positive & 2 & Singleton \\
\hline & \multirow[t]{2}{*}{7} & \multirow[t]{2}{*}{ Pseudomonas } & Negative & 1 & Singleton \\
\hline & & & Positive & 2 & Singleton \\
\hline & \multirow[t]{2}{*}{8} & \multirow[t]{2}{*}{ Staphylococcus } & Negative & 1 & Singleton \\
\hline & & & Positive & 2 & Singleton \\
\hline & \multirow[t]{2}{*}{9} & \multirow[t]{2}{*}{ Family history } & Negative & 1 & Singleton \\
\hline & & & Positive & 2 & Singleton \\
\hline & \multirow[t]{2}{*}{10} & \multirow[t]{2}{*}{ Abnormal stool } & Negative & 1 & Singleton \\
\hline & & & Positive & 2 & Singleton \\
\hline \multirow[t]{2}{*}{ Output } & \multirow[t]{2}{*}{1} & \multirow[t]{2}{*}{ CF diagnosis } & Non-CF & 1 & Singleton \\
\hline & & & $\mathrm{CF}$ & 2 & Singleton \\
\hline
\end{tabular}




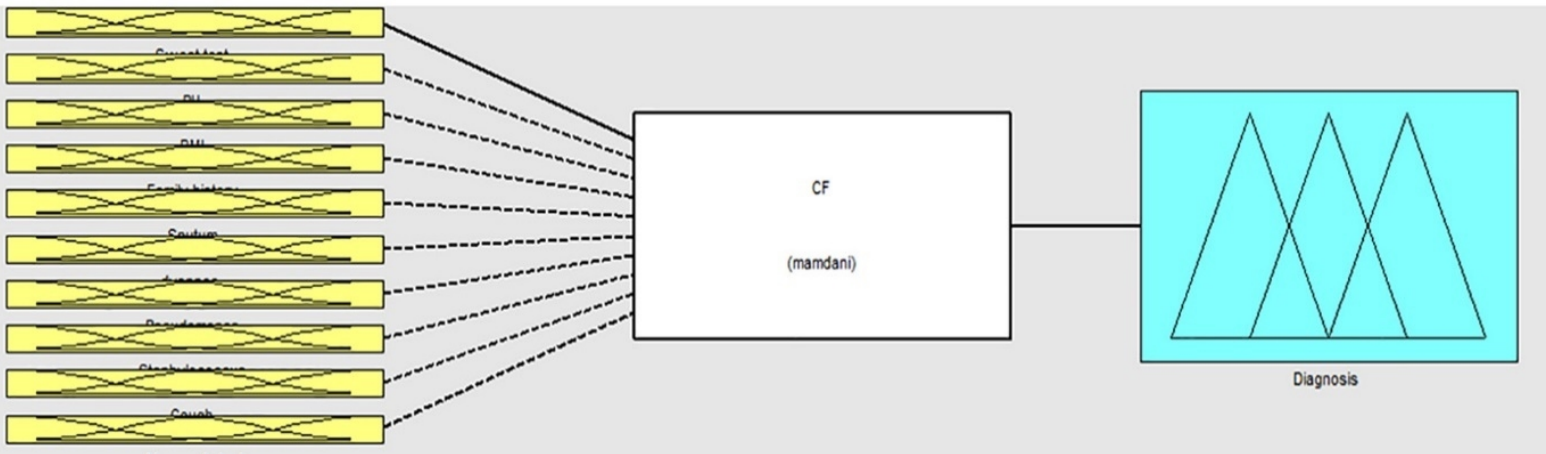

Figure 2. Fuzzy inference system block diagram

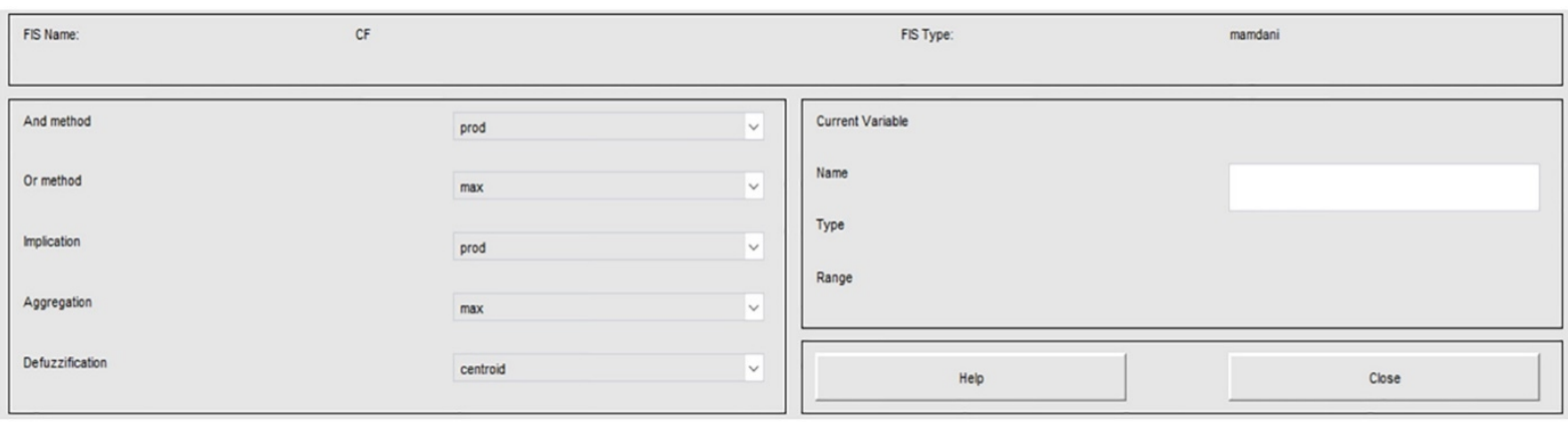

Figure 3. Constructed FIS system characteristics

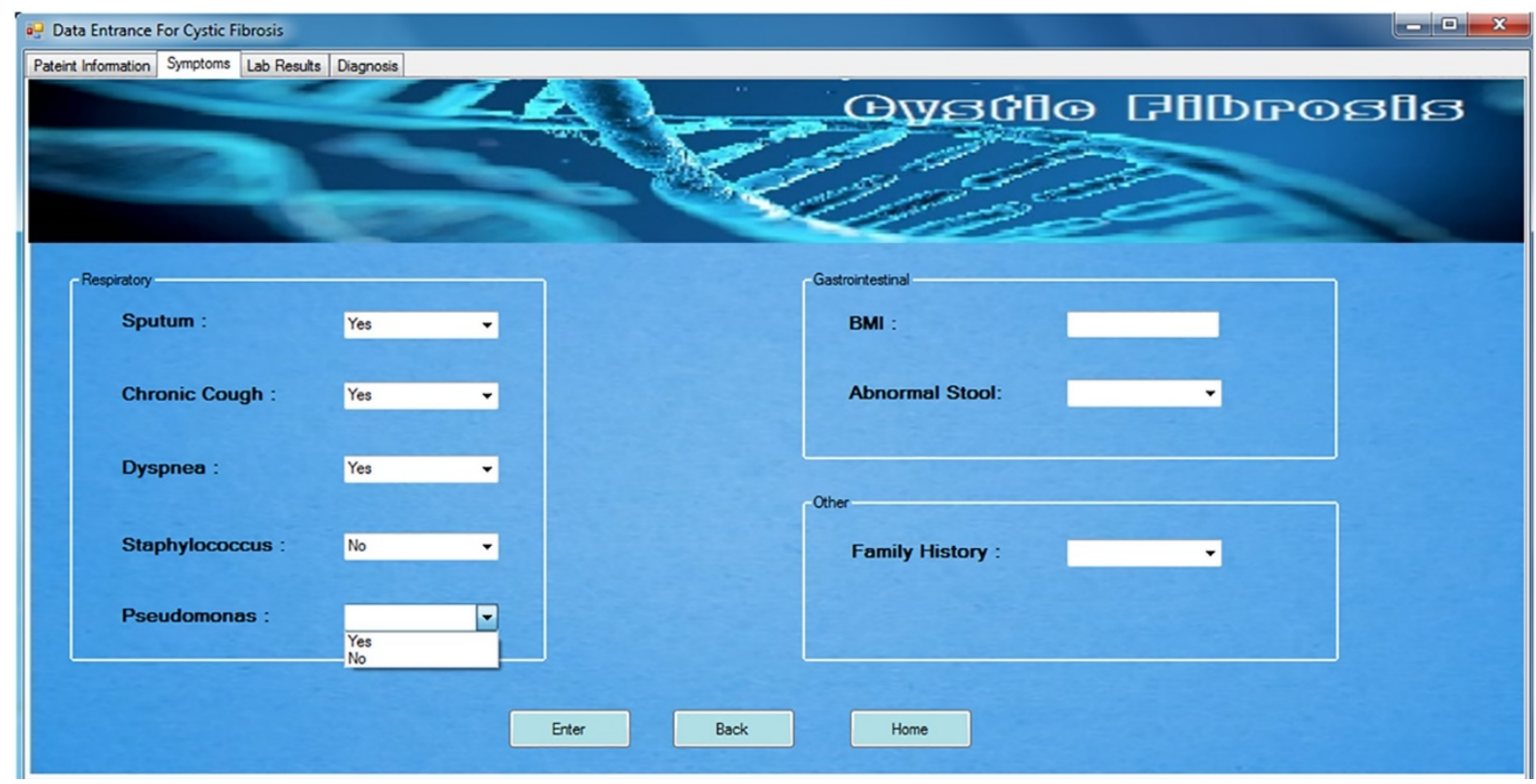

Figure 4. Graphical user interface (GUI)

\subsection{Evaluation}

To evaluate the performance of the developed fuzzy expert system, a list of medical records of newly diagnosed CF patients was reviewed during August 2016 to January 2017. The patients were serially admitted to the CF Clinic in the Pediatric Respiratory Diseases Center (National Research Institute of Tuberculosis and Lung Diseases (NRITLD), Masih Daneshvari Hospital, Tehran, Iran) which is the CF reference center in Iran. All the procedures of the study were approved by the ethics committee of Shahid Beheshti Medical Sciences University, which was fully in accordance with the Declaration of Helsinki Ethical Principles for Medical Research Involving Human Subjects [Ethics code: IR.SBMU.NRITLD.REC.1395.260]. Although based on Cochran's Formula, sample volume in this 
research estimated 96 people to a safety level of $90 \%$. Due to the lack of comprehensive medical records containing all the mentioned variables in this paper, the sample of patients' data for testing this system was restricted to 70 patients (42 CF , 28 non-CF) referring to Pediatric Respiratory Diseases Center (Masih Daneshvari Hospital), and they were examined by pediatric respiratory diseases physicians. Then, participants' data were analyzed to compare the outcomes of the system, and the results recorded in medical records. Finally, based on the analyzed data, the accuracy, specificity, and sensitivity of the predictive model were determined.

\section{Results}

Figure 5 demonstrates the fuzzy sets related to the linguistic variables sweat test, PH and BMI; and based on the domain knowledge, the numbers of membership functions are defined by the experts. In order to evaluate FIS, the performance measures such as precision, specificity, sensitivity, F1-measure and accuracy have been used which are calculated by the following equations:

Precision or Positive Predictive Value $(\mathrm{PPV})=\mathrm{TP} /(\mathrm{TP}+\mathrm{FP})$

Specificity $(\mathrm{SPC})$ or true negative rate $=\mathrm{TN} / \mathrm{N}=\mathrm{TN} /(\mathrm{TN}+\mathrm{FP})$

Sensitivity or True Positive Rate $(\mathrm{TPR})$ or recall $=\mathrm{TP} / \mathrm{P}=\mathrm{TP} /(\mathrm{TP}+\mathrm{FN})$

Accuracy $(\mathrm{ACC})=(\mathrm{TP}+\mathrm{TN}) /(\mathrm{TP}+\mathrm{TN}+\mathrm{FP}+\mathrm{FN})$

$\mathrm{F} 1-$ measure $=2 *($ precision $*$ recall $) /($ precision + recall $)$

Where TP, FN, FP and TN are true positive, false negative, false positive and true negative, respectively. Figure 6 exhibits the desired output and the actual output of the CF-FIS model. It can be observed that the constructed system performance is good in diagnosis of CF. A confusion matrix includes information concerning real and predicted classifications done by a classification system. Due to the $n$ classes a confusion matrix is an $n \times n$ matrix. Performance of classification systems is usually evaluated using the data in the matrix. The confusion matrix of the CF-diagnosis fuzzy system is displayed in Table 4 which elaborates the strengths and weaknesses of this system. For below confusion matrix, true positives for class $=$ ' $\mathrm{CF}$ ' is 40 , while false positive is 3 for class $=$ 'non-CF', true negative is 25 and false negative is 2 i.e. diagonal elements of matrix $25+40=65$ represent the right instances classified and next elements $2+3=5$ show the incorrect instances. Regarding the system performance in terms of all five metrics including sensitivity, specificity, precision, accuracy and F1-measure, it can be mentioned that sensitivity had the maximum rate with about 0.95 and specificity had the minimum rate with a bit more than 0.89 , while $\mathrm{F} 1$-measure, precision and accuracy were respectively $0.94,0.93$, and almost 0.93 . Figure 7 shows ROC curve comparing the sensitivity and specificity of the suggested system (AUC=Area Under Curve).
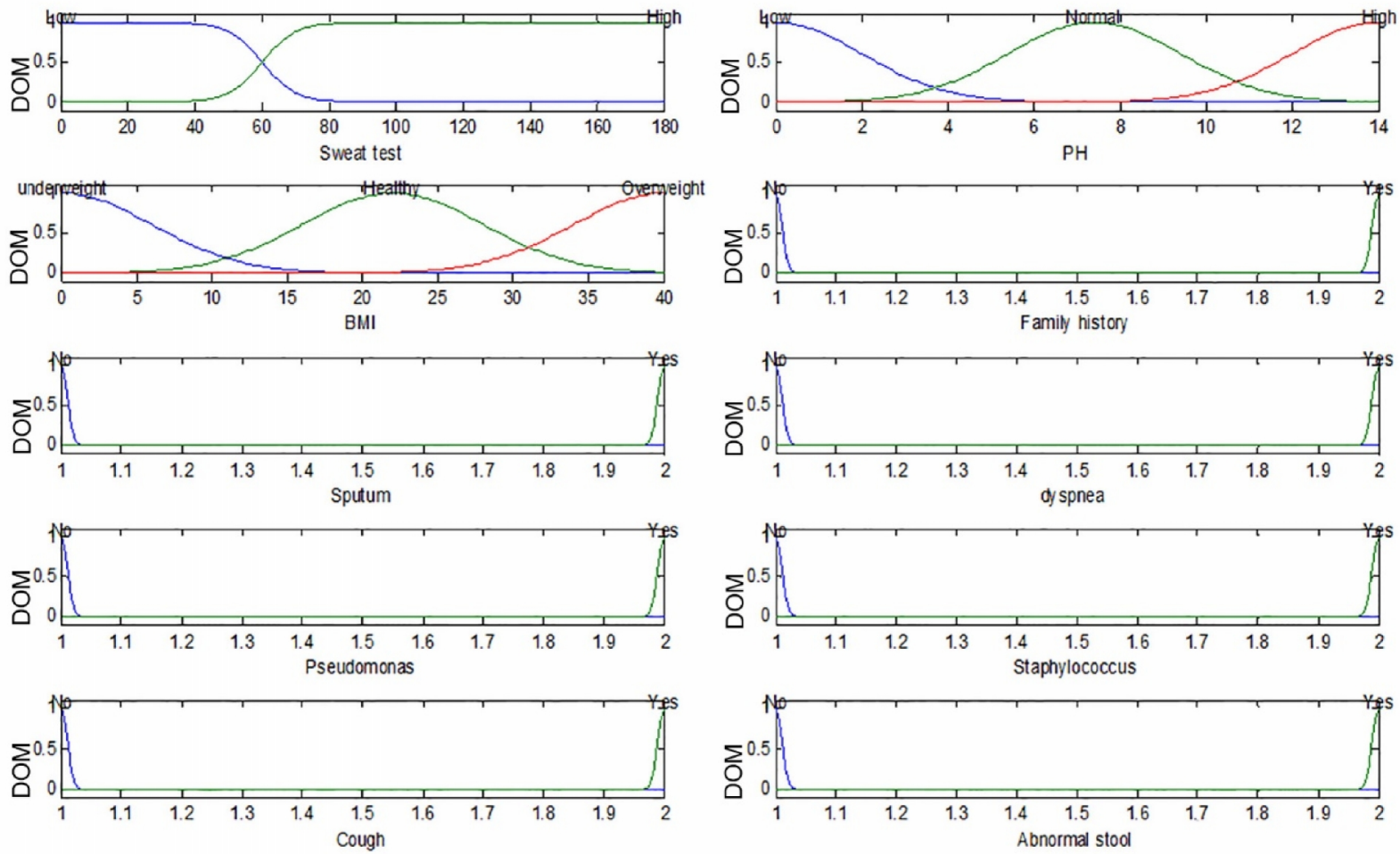

Figure 5. Membership functions for input/output variables of the suggested system. DOM: degree of membership 


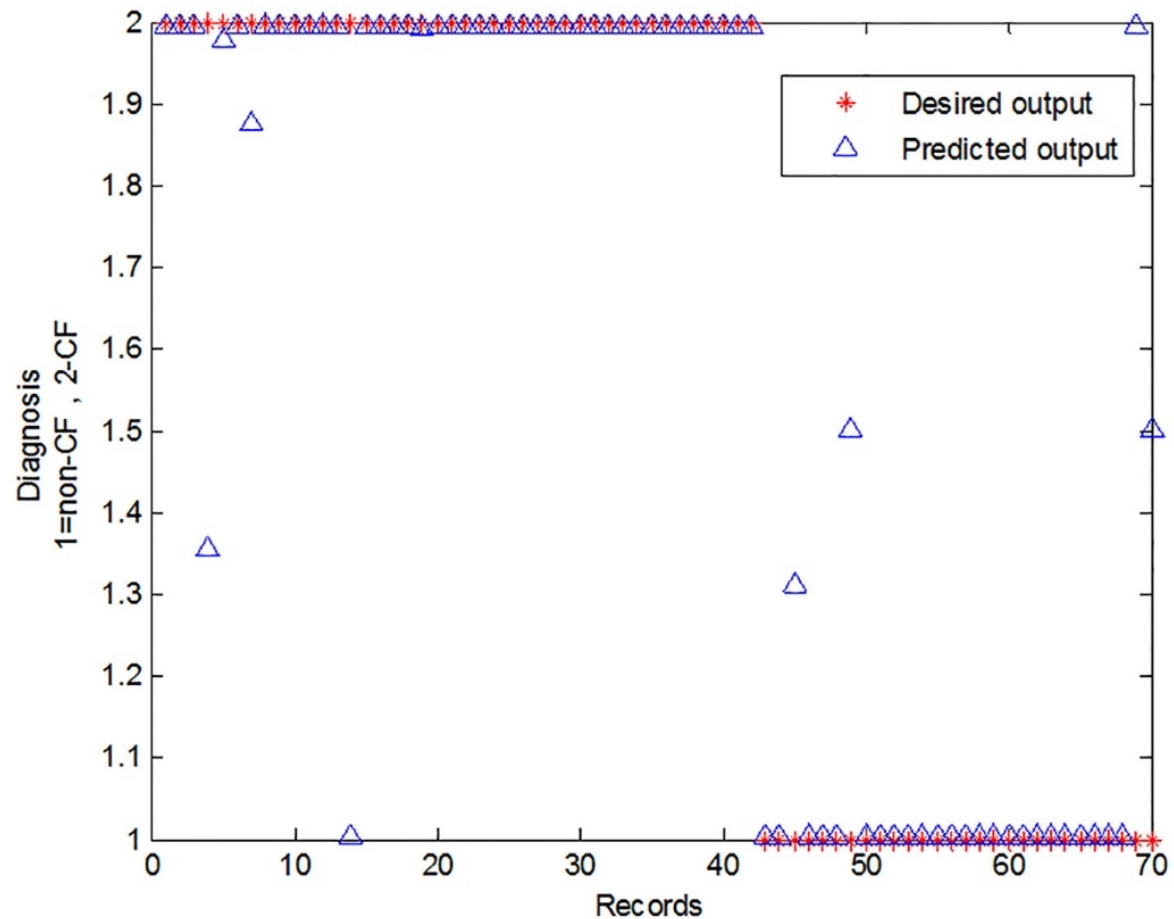

Figure 6. System output and desired output.

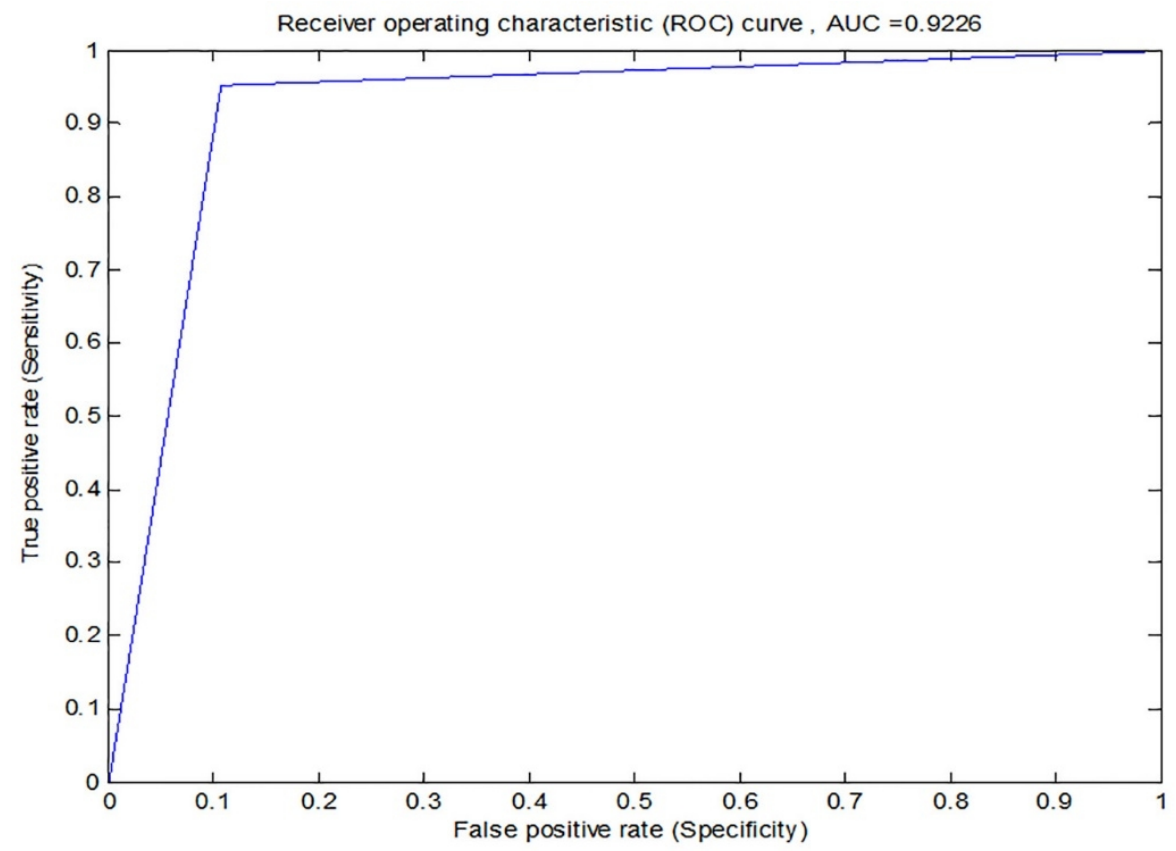

Figure 7. ROC curve of the proposed CF-FIS model

Table 4. Confusion matrix for the CF-diagnosis fuzzy system

\begin{tabular}{|l|l|l|l|}
\hline \multicolumn{2}{|c|}{} & \multicolumn{2}{|l|}{ Predicted } \\
\cline { 3 - 4 } \multicolumn{2}{|c|}{} & Non-CF & CF \\
\hline Desired / actual & Non-CF & 25 & 3 \\
\cline { 2 - 4 } & CF & 2 & 40 \\
\hline
\end{tabular}




\section{Discussion}

In the present paper, the suggested design and evaluation of a fuzzy expert system for diagnosis of CF are represented. The diagnosis process was designed based on the expert's knowledge and existing literature. In the real world, physicians use the patient's risk factors and symptoms as input and then based on which and using their own experiences, proper diagnosis is prescribed for each patient. Hence, given the expert systems approach within this system, the CF risk factors and symptoms saved in the working memory and knowledge base include some rules from the domain expert. Then, for eliminating the uncertainty in the problem, fuzzy method is used to make a valid decision for each patient. Moreover, by user interface, a good relationship between user and the system is provided. The comparison between physicians' evaluations and the system's results (for these 70 patients) confirms the efficacy of this system. Therefore, the results of this study are in compliance with other researches. In the study of Zolnoori et al., the system reinforced a good efficiency of the fuzzy expert system for predicting the possibility of fatal asthma (28). While in the study of Avci, the classification accuracy of this GDA-LS-SVM expert system was acquired about $96.875 \%$ from the outcomes of these experimental studies (30). The qualified results of Zolnoori et al. have been compared with physicians' evaluations. The obtained results confirmed the complete accordance between system's results and physicians' evaluations. In addition, the system represented quantified results in gradation that reflected a slight difference between degrees of asthma exacerbation (31). Evaluating the performance of the Zolnoori et al. system comprising 28 asthmatic patients, reinforced that the system's results not only corresponded to the evaluations of physicians, but also represented the slight differences of asthmatic patients placed in the specific category introduced by the guidelines (33). The findings of Zolnoori et al. showed that their system met the requirements of user interaction satisfaction (34). The system efficiency of Vasheghani Farahani et al. was appraised by 60 CT scans gathered by Lung Image Database Consortium (LIDC), and the findings represented good progression in diagnosing pulmonary nodules (36). Yang et al. presented that using fuzzy expert systems was a viable solution for QA of toxicological data (42). The results not only showed the potential of approach in automatizing the quality evaluation processes, but they also posed some questions which led to further research (42). The obtained results from the system reveal that the diagnostic system is giving expected results and its efficacy is confirmed by the specialist physician in this field. The proposed system was not intended to replace the specialist doctor; nevertheless, it can be used to help a general practitioner or specialist doctor in diagnosing and predicting the patient's situation. The defined rules in the expert system indeed replicate the kind of decision making done in the trained mind of a specialist. The importance of the developed Fuzzy Expert System (FES) lies in the fact that management of CF depends fully on the severity of the disease and it requires the knowledge and experience of a pediatrician/respirologist to give a correct diagnosis regarding the severity of $\mathrm{CF}$, so the proposed expert system enables the less experienced junior physicians to achieve a better diagnosis, as it holds the expert knowledge in an intelligent system to be utilized effectively by others. The provided rules in the design of the fuzzy expert system are not exhaustive; nonetheless the system gives a good insight about different pulmonary diseases by predicting their severity almost equivalent to that of a medical expert. These preliminary results suggest that fuzzy relations should be considered as a good method to provide valid diagnoses and decision-making procedures in pediatrics. Increasing the sample size in future studies could be fruitful in evaluating the performance of this system. In order to improve system accuracy in a primary care setting (without laboratory data), variables can be restricted to symptoms data and historical data.

\section{Conclusions}

In general, it can be said that the studies on the use of such FES in finding medical diagnosis are very promising for the future. An FES can add valued input if incorporated into a routine clinical consultation, but it could never replace the role of clinicians. The expert system is a supportive tool in the treatment of cystic fibrosis, but the final decision can only be made by the specialist. However, in a decision-making process, the tools and techniques like fuzzy logic are as useful as the specialist's diagnosis. As far as the data are trusty, the system will be able to make a diagnostic offer that assists the specialist to provide the patients with suitable treatment. In summary, despite the system showing complete positive correlation with specialists, further tests are necessary to determine the error percentage of the system. This system can be considered as a complimentary instrument for specialists, and it is not intended as a substitute for their own experiences. Only the specialists can understand the individual criteria and deliberations of each patient and based on their own expertise, only they can make the final decisions. It should be mentioned however, that as well as being a supportive tool, the system in which the information, knowledge, and expertise from certified specialists is stored, can also be used effectively as a training tool for new physicians.

\section{Acknowledgments:}

The authors would like to thank National Research Institute of Tuberculosis and Lung Diseases (NRITLD), Masih Daneshvari Hospital, Shahid Beheshti University of Medical Sciences for supporting this project. 


\section{Conflict of Interest:}

There is no conflict of interest to be declared.

\section{Authors' contributions:}

All authors contributed to this project and article equally. All authors read and approved the final manuscript.

\section{References:}

1) Josefiok M, Sauer J. Towards an Expert System for the Field of Neurology Based on Fuzzy Logic. Joint German/Austrian Conference on Artificial Intelligence (Künstliche Intelligenz). 2015; 333-40. doi: 10.1007/978-3-319-24489-1_31.

2) Kunhimangalam R, Ovallath S, Joseph PK. A novel fuzzy expert system for the identification of severity of carpal tunnel syndrome. BioMed research international. 2013; 2013. doi: 10.1155/2013/846780.

3) Elahi E, Khodadad A, Kupershmidt I, Ghasemi F, Alinasab B, Naghizadeh R, et al. A haplotype framework for cystic fibrosis mutations in Iran. J Mol Diagn. 2006; 8(1): 119-27. doi: 10.2353/jmoldx.2006.050063. PMID: 16436643, PMCID: PMC1867567.

4) Singh M, Rebordosa C, Bernholz J, Sharma N. Epidemiology and genetics of cystic fibrosis in Asia: In preparation for the next-generation treatments. Respirology. 2015; 20(8): 1172-81. doi: 10.1111/resp.12656. PMID: 26437683.

5) Farjadian S, Moghtaderi M, Kashef S, Alyasin S, Najib K, Saki F. Clinical and genetic features in patients with cystic fibrosis in southwestern Iran. Iran J Pediatr. 2013; 23(2): 212-5. PMID: 23724185, PMCID: PMC3663315.

6) Baghaie N, Kalilzadeh S, Hassanzad M, Parsanejad N, Velayati A. Determination of mortality from cystic fibrosis. Pneumologia. 2010; 59(3): 170-3. PMID: 21053647.

7) Kahkouee S, Namini AK, Boloursaz MR. Quantitative evaluation of high-resolution CT findings in advanced cystic fibrosis patients based on the Brody scoring. Journal of Comprehensive Pediatrics. 2014; 5(1). doi: 10.17795/compreped-4901.

8) Mehdizadeh Hakkak A, Keramatipour M, Talebi S, Brook A, Tavakol Afshari J, Raazi A, et al. Analysis of CFTR gene mutations in children with cystic fibrosis, first report from North-East of Iran. Iran J Basic Med Sci. 2013; 16(8): 917-21. PMID: 24106596, PMCID: PMC3786104.

9) Khalilzadeh S, Boloursaz M, Baghaie N, Fard EH, Hassanzad M, Emami H. Microbial colonization and drug resistance in patients with cystic fibrosis. Journal of Comprehensive Pediatrics. 2012; 3(1): 25-8. doi: 10.17795/compreped-6944.

10) Khalilzadeh S, Hassanzad M, Baghaie N, Parsanejad N, Boloursaz MR, Fahimi F. Shwachman score in clinical evaluation of cystic fibrosis. Journal of Comprehensive Pediatrics. 2013; 4(1): 82-5. doi: 10.17795/compreped-4558.

11) Khalilzadeh S, Kahkouee S, Hassanzad M, Parsanejad N, Baghaie N, Bloorsa MR. The correlation of brody high resolution computed tomography scoring system with clinical status and pulmonary function test in patients with cystic fibrosis. Iran J Med Sci. 2011; 36(1): 18-23. PMID: 23365473, PMCID: PMC3559112.

12) Vasheghani Farahani F, Zarandi MF, Ahmadi A. Fuzzy rule based expert system for diagnosis of lung cancer. Fuzzy Information Processing Society (NAFIPS) held jointly with 2015 5th World Conference on Soft Computing, 2015 Annual Conference of the North American; 2015: IEEE. doi: 10.1109/NAFIPSWConSC.2015.7284206.

13) Alipour J, Safari Lafti S, Askari Majdabadi H, Yazdiyani A, Valinejadi A. Factors affecting hospital information system acceptance by caregivers of educational hospitals based on technology acceptance model (TAM): A study in Iran. IIOAB Journal. 2016; 119-23.

14) Norouzi J, Yadollahpour A, Mirbagheri SA, Mazdeh MM, Hosseini SA. Predicting renal failure progression in chronic kidney disease using integrated intelligent fuzzy expert system. Computational and mathematical methods in medicine. 2016; 2016. doi: 10.1155/2016/6080814.

15) Reis MA, Ortega NR, Silveira PS. Fuzzy expert system in the prediction of neonatal resuscitation. Braz J Med Biol Res. 2004; 37(5): 755-64. doi: 10.1590/S0100-879X2004000500018. PMID: 15107939.

16) Neshat M, Yaghobi M, Naghibi M, Esmaelzadeh A. Fuzzy expert system design for diagnosis of liver disorders. Knowledge Acquisition and Modeling, 2008 KAM'08 International Symposium on; 2008: IEEE. doi: 10.1109/KAM.2008.43.

17) Zarandi MF, Zarinbal M, Izadi M. Systematic image processing for diagnosing brain tumors: A Type-II fuzzy expert system approach. Applied soft computing. 2011; 11(1): 285-94. doi: 10.1016/j.asoc.2009.11.019. 
18) Nobile L, Cosenza B, Amato M, Guarnotta V, Giordano C, Galluzzo A, et al. Development of a fuzzy expert system for the control of glycemia in type 1 diabetic patients. Comput Aided Chem Eng. 2011; 29: 1568-72. doi: 10.1016/B978-0-444-54298-4.50092-1.

19) Mago VK, Mago A, Sharma P, Mago J. Fuzzy logic based expert system for the treatment of mobile tooth. Adv Exp Med Biol. 2011; 696: 607-14. doi: 10.1007/978-1-4419-7046-6_62. PMID: 21431602.

20) Keles A, Keles A, Yavuz U. Expert system based on neural-fuzzy rules for thyroid diseases diagnosis. Computer Applications for Bio-technology, Multimedia, and Ubiquitous City. 2012; 94-105.

21) Ephzibah E, Sundarapandian V. A Fuzzy Rule Based Expert System for Effective Heart Disease Diagnosis. Advances in Computer Science and Information Technology Computer Science and Engineering. 2012: 196203. doi: 10.1007/978-3-642-27308-7_20.

22) Domínguez Hernández KR, Aguilar Lasserre AA, Posada Gómez R, Palet Guzmán JA, González Sánchez BE. Development of an expert system as a diagnostic support of cervical cancer in atypical glandular cells, based on fuzzy logics and image interpretation. Computational and mathematical methods in medicine. 2013. doi: 10.1155/2013/796387. PMID: 23690881, PMCID: PMC3652118.

23) Zarandi MF, Damirchi-Darasi SR, Izadi M, Turksen I, Ghahazi MA. Fuzzy rule based expert system to diagnose spinal cord disorders. Norbert Wiener in the 21st Century (21CW), 2014 IEEE Conference on; 2014: IEEE. doi: 10.1109/NORBERT.2014.6893897.

24) Ghahazi MA, Zarandi MF, Harirchian M, Damirchi-Darasi SR. Fuzzy rule based expert system for diagnosis of multiple sclerosis. Norbert Wiener in the 21st Century (21CW), 2014 IEEE Conference on; 2014: IEEE. doi: 10.1109/NORBERT.2014.6893855.

25) Zarinbal M, Zarandi MF, Turksen IB, Izadi M. A type-2 fuzzy image processing expert system for diagnosing brain tumors. J Med Syst. 2015; 39(10): 110. doi: 10.1007/s10916-015-0311-6. PMID: 26276018.

26) Chakraborty C, Mitra T, Mukherjee A, Ray AK. CAIDSA: Computer-aided intelligent diagnostic system for bronchial asthma. Expert Systems with Applications. 2009; 36(3): 4958-66. doi: 10.1016/j.eswa.2008.06.025.

27) Zarandi MF, Zolnoori M, Moin M, Heidarnejad H. A fuzzy rule-based expert system for diagnosing asthma. Scientia Iranica Transaction E, Industrial Engineering. 2010; 17(2): 129.

28) Zolnoori M, Zarandi MHF, Moin M, Taherian M. Fuzzy rule-based expert system for evaluating level of asthma control. J Med Syst. 2012; 36(5): 2947-58. doi: 10.1007/s10916-011-9773-3. PMID: 21912973.

29) Lomotan EA, Hoeksema LJ, Edmonds DE, Ramírez-Garnica G, Shiffman RN, Horwitz LI. Evaluating the use of a computerized clinical decision support system for asthma by pediatric pulmonologists. Int $\mathrm{J}$ Med Inform. 2012; 81(3): 157-65. doi: 10.1016/j.ijmedinf.2011.11.004. PMID: 22204897, PMCID: PMC3279612.

30) Avci E. A new expert system for diagnosis of lung cancer: GDA_LS_SVM. J Med Syst. 2012; 36(3): 20059. doi: 10.1007/s10916-011-9660-y. PMID: 21340704.

31) Zolnoori M, Zarandi MH, Moin M. Application of intelligent systems in asthma disease: designing a fuzzy rule-based system for evaluating level of asthma exacerbation. J Med Syst. 2012; 36(4): 2071-83. doi: 10.1007/s10916-011-9671-8. PMID: 21399914.

32) Dexheimer JW, Abramo TJ, Arnold DH, Johnson KB, Shyr Y, Ye F, et al. An asthma management system in a pediatric emergency department. Int $\mathrm{J}$ Med Inform. 2013; 82(4): 230-8. doi: 10.1016/j.ijmedinf.2012.11.006. PMID: 23218449, PMCID: PMC3646328.

33) Zolnoori M, Zarandi MH, Moin M, Teimorian S. Fuzzy rule-based expert system for assessment severity of asthma. J Med Syst. 2012; 36(3): 1707-17. doi: 10.1007/s10916-010-9631-8. PMID: 21128097.

34) Zolnoori M, Jones JF, Moin M, Heidarnejad H, Fazlollahi MR, Hosseini M. Evaluation of user interface of computer application developed for screening pediatric asthma. International Conference on Universal Access in Human-Computer Interaction. 2013; 563-70. doi: 10.1007/978-3-642-39194-1_65.

35) Anand SK, Kalpana R, Vijayalakshmi S. Design and implementation of a fuzzy expert system for detecting and estimating the level of asthma and chronic obstructive pulmonary disease. Middle-East Journal of Scientific Research. 2013; 14(11): 1435-44. doi: 10.5829/idosi.wasj.2013.23.02.13046.

36) Vasheghani Farahani F, Ahmadi A, Zarandi MF. Lung nodule diagnosis from CT images based on ensemble learning. Computational Intelligence in Bioinformatics and Computational Biology (CIBCB), 2015 IEEE Conference on; 2015: IEEE.

37) Efosa IC, Akwukwuma V. Knowledge-based fuzzy inference system for sepsis diagnosis. International Journal of Computational Science and Information Technology (IJCSITY). 2013; 1(3): 1-7. doi: 10.5121/ijcsity.2013.1301. 
38) Garibaldi JM. Fuzzy expert systems. Do Smart Adaptive Systems Exist? 2005; 105-32. doi: 10.1007/3-54032374-0_6.

39) Nascimento LF, Rocha Rizol PM, Abiuzi LB. Establishing the risk of neonatal mortality using a fuzzy predictive model. Cad Saude Publica. 2009; 25(9): 2043-52. doi: 10.1590/S0102-311X2009000900018. PMID: 19750391.

40) Adeli A, Neshat M. A fuzzy expert system for heart disease diagnosis. Proceedings of International Multi Conference of Engineers and Computer Scientists Hong Kong: IMECS Conference Proceedings; 2010.

41) Wang L-X. A course in fuzzy systems: Prentice-Hall press, USA; 1999.

42) Yang L, Neagu D, Cronin MT, Hewitt M, Enoch SJ, Madden JC, et al. Towards a fuzzy expert system on toxicological data quality assessment. Molecular Informatics. 2013; 32(1): 65-78. doi: 10.1002/minf.201200082. PMID: 27481024. 